\title{
Recognizing systemic sclerosis: comparative analysis of various sets of classification criteria
}

\author{
Katarzyna Romanowska-Próchnicka ${ }^{1,2}$, Marcela Walczyk², Marzena Olesińska ${ }^{2}$ \\ ${ }^{1}$ Department of General and Experimental Pathology, $2^{\text {nd }}$ Faculty of Medicine, Medical University of Warsaw, Poland \\ ${ }^{2}$ Department of Systemic Connective Tissue Diseases, National Institute of Geriatrics, Rheumatology and Rehabilitation, Warsaw, \\ Poland
}

\begin{abstract}
Systemic sclerosis is a complex disease characterized by autoimmunity, vasculopathy and tissue fibrosis. Although most patients present with some degree of skin sclerosis, which is a distinguishing hallmark, the clinical presentation vary greatly complicating the diagnosis. In this regard, new classification criteria were jointly published in 2013 by American College of Rheumatology (ACR) and European League Against Rheumatism (EULAR). A recent major development in the classification criteria is improved sensitivity, particularly for detecting early disease. The new criteria allow more cases to be classified as having systemic sclerosis (SSc), which leads to earlier treatment. Moreover it is clinically beneficial in preventing the disease progression with its irreversible fibrosis and organ damage. The aim of this review is to give insight into new classification criteria and current trends in the diagnosis of systemic sclerosis.
\end{abstract}

Key words: systemic sclerosis, new classification ACR/EULAR criteria 2013, 1980 ACR classification criteria.

\section{Introduction}

Systemic sclerosis (SSc; scleroderma) is a heterogeneous, autoimmune disease characterized by fibroblast dysfunction, vasculopathy and dysregulation of the immune system. This mechanism causes vascular ischemia, followed by an immunologic response and eventually fibrosis.

Clinical manifestation of scleroderma can vary greatly making both diagnosis and further treatment difficult and complicated. For example, characteristic tightening of the skin can range from none to all. The severity of the disease may vary from puffy fingers in mild involvement to the hardest involvement where all parts of limbs, face, and the chest are affected.

A major achievement of 2013 has been the validation of new classification ACR/EULAR American College of Rheumatology/European League Against Rheumatism criteria, allowing earlier diagnosis and earlier treatment of SSc.
A major problem with the previous one, 1980 ACR classification criteria was low sensitivity, particularly in cases of early SSc and limited cutaneous SSc (IcSSC). Its sensitivity and specificity in the validation sample were 0.75 and 0.72 , respectively [1].

In joint effort for greater sensitivity the EULAR and ACR rheumatologists initiated a revision of the classification criteria. It was a long process of choosing clinical and laboratory features. Finally, Delphi method and nominal group technique of 8 criteria were developed succeeding the initial approach, with a different weight assigned to each criterion. According to this new technique patients achieving a score of 9 or more are classified as having SSc [1]. These new criteria reflect a major, international effort of 44 investigators, and enabled collecting data of over 1150 systemic sclerosis cases and over 1300 controls [1]. The New ACR/EULAR criteria are not to be applied when another explanation for a patient's signs and symptoms exists and if there is skin fibrosis 
Table I. The ACR-EULAR Criteria for the classification of systemic sclerosis [1]

\begin{tabular}{|c|}
\hline 1. These criteria are applicable to any patient considered for inclusion in a SSc study \\
\hline 2. These criteria are not applicable to: \\
\hline $\begin{array}{l}\text { Patients having a SSc-like disorder better explaining their manifestations, such as: nephrogenic sclerosing fibrosis, general- } \\
\text { ized morphea, eosinophilic fasciitis, scleredema diabeticorum, scleromyxedema, erythromyalgia, porphyria, lichen sclerosis, } \\
\text { graft versus host disease, and diabetic cheiropathy }\end{array}$ \\
\hline Patients with "Skin thickening sparing the fingers" \\
\hline Items Sub-items Weight/Score \\
\hline Skin thickening of the fingers of both hands extending proximal to the metacarpophalangeal joints (sufficient criterion) 9 pts. \\
\hline Skin thickening of the fingers^ (only the highest score counts) \\
\hline Puffy fingers 2 pts. \\
\hline Sclerodactyly of the fingers (distal to MCP but proximal to the PIPs) 4 pts. \\
\hline Finger tip lesions^ (only the highest score counts) \\
\hline Digital tip ulcers 2 pts. \\
\hline Finger tip pitting scars 3 pts. \\
\hline Telangiectasia 2 pts. \\
\hline Abnormal nailfold capillaries 2 pts. \\
\hline Pulmonary arterial hypertension and/or interstitial lung disease* ${ }^{*}$ maximum score is 2$)$ \\
\hline Raynaud's phenomenon 3 pts. \\
\hline $\begin{array}{l}\text { Scleroderma related antibodies** (any of anti-centromere, anti-topoisomerase, [anti-Scl 70], anti-RNA polymerase III) } 3 \text { pts. } \\
\left({ }^{* \star} \text { maximum score is 3) }\right.\end{array}$ \\
\hline
\end{tabular}

TOTAL SCORE^: Patients having a total score of 9 or more are being classified as having definite systemic sclerosis.

PAH is pulmonary arterial hypertension. The definition is proven PAH by right heart catheterization. ILD is interstitial lung disease defined as pulmonary fibrosis on HRCT or chest radiograph, most pronounced in the basilar portions of the lungs, or presence of 'velcro' crackles on auscultation not due to another cause such as congestive heart failure. ^ Add the maximum weight (score) in each category to calculate the total score.

without ever having finger involvement [1]. The new SSc classification criteria are based on the 9 points system and include hands skin thickening/puffy fingers, digit lesions, Raynaud's phenomenon (RP), abnormal nail-fold capillaries, teleangiectasia, lung diseases (pulmonary arterial hypertension - PAH or interstitial lung disease - ILD), and SSc-related autoantibodies (anti-centromere, anti-topoisomerase I and anti-RNA polymerase III) (Table I). Its sensitivity and specificity in the validation sample were, 0.91 and 0.92 respectively [1].

Many studies were devoted to comparative analysis of old and new criteria. The biggest differences in diagnosis were noticed in mild and early group of SSc. In VEDOSS cohort, 242/304 (79.6\%) patients met the ACR/ EULAR criteria compared to $162 / 304$ (53.3\%) patients who met the 1980 ACR criteria [2].

Additionally, the fact that the latest classification criteria can identify early disease was shown in one of the biggest study populations with scleroderma (the Canadian Scleroderma Research Group) [3]. In a study of 724 SSc patients new criteria have been met in $98.3 \%$ vs. $88.3 \%$ of patients diagnosed according to old ones. Compared to the old system, the new criteria were also more sensitive in subgroups of patients with early $(<2$ years) SSc (98.7\%, 84.7\% respectively). Moreover, cases of patients with lcSSc (98.8\%, 85.6\%, respectively), anticentromere antibodies (98.9\%, 79.8\%, respectively), or those with no skin involvement proximally to the metacarpophalangeal joints (97\%, 60\% respectively) additionally confirmed that the new classification can accurately define scleroderma [3].

Despite the abovementioned advantages, since their publication the new classification criteria have been also a subject of controversy. First aspect is related to the differences between classification and diagnostic criteria [4]. Scleroderma has no diagnostic test for disease recognition. Diagnostic criteria are intended to show features of the disease that characterize most cases and cover the full spectrum of the disease. Classification criteria, however, are designed to identify a well-characterized group of patients within the spectrum of the disease. Classification criteria inform us about concept of the disease. Many characteristics like prevalence of similar diseases, geography, ethnic origin and race can affect the degree of overlapping the two classifications [4].

Another controversy is connected with its complexity of calculation. There are 8 different criteria with numeric additive system, which is still to many for critics. On the other hand reducing and simplifying the criteria would result in a loss of sensitivity and specificity so 
important in the case. There are also different opinions when it comes to add individuals with mixed connective tissue disease (MCTD) to scleroderma group. The 1980 ACR criteria exclude them from that group, although about one third of MCTD patients are SSc-predominant. Last controversial aspect is related to juvenile systemic sclerosis. The ACR/EULAR criteria should be tested for validity in juvenile systemic sclerosis [4].

\section{Previous criteria}

Over time, several different criteria for classification of SSc have been proposed. To date, the most widely accepted were the ones published by American Rheumatism Association (ARA) in 1980 [5]. They were validated in a large population of patients and for more than 30 years have been commonly used. The 1980 ARA set of criteria were proposed as "preliminary". The idea was to create criteria with high specificity to minimize the risk of false positive diagnoses. The authors emphasized that the aim of the classification was not to support the early diagnosis of SSc but rather to gather subjects with the same clinical entity to facilitate the research studies. An important disadvantage was the loss of sensitivity. This led to exclusion of a significant number of SSc subjects, especially early and mild cases, as well as the patients with the limited subtypes. Subsequently these groups of patients were disqualified from participating in clinical investigation and therapeutic trials. The ARA 1980 criteria include skin thickening proximal to the metacarpophalangeal joints which is a major and sufficient criterion as well as 3 minor criteria (1) sclerodactyly, (2) digital pitting scars of fingertips of distal finger, (3) bibasilar pulmonary fibrosis (at least 2 of 3 items have to be achieved for SSc diagnosis [5]).

This approach focuses on the fibrotic manifestations of the disease. Taking into consideration the other $2 \mathrm{ma}$ jor SSc features - vasculopathy and immune abnormalities, which were described after publication of the criteria in 1980, Lonzetti proposed updating the ARA criteria by adding the nailfold capillaroscopy abnormalities and SSc-specific antibodies [5].

Thereafter, another modification was proposed by Nadashkevich but despite the improved sensitivity and specificity the alternative classification has not gained a lot of popularity and was not externally validated [6]. Subsequently, to meet the need for identifying the SSc patients at an earlier stage LeRoy and Medsger suggested an alternative classification [7]. Finally, considering the risk of internal organs involvement in patients at early stage of SSc, who do not fulfil the 1980 criteria, through collective effort of EUSTAR (The European Scleroderma Trials and Research group) the preliminary criteria for the very early diagnosis of SSc were developed (VEDOSS) [2, 8].

\section{Skin thickening of the fingers of both hands extending proximal to the metacarpophalangeal joints (sufficient criterion) -9 pts.}

Several studies demonstrate that skin thickness score is a prediction factor in scleroderma and correlates with the outcome in systemic sclerosis $[9,10]$. Also change in skin score involves visceral complications. There is an association between severe skin involvement and burden of organ-based complications. Death or major organ based morbidity occurs in 50\% of dcSSc cases within 3 years of disease onset. A fully validated method ('gold standard') for measuring the dermal skin thickness is the modified Rodnan skin score (mRSS). mRSS is considered the most appropriate and reproducible technique for measuring skin involvement using palpation in SSc [11]. The mRSS requires some experience, a careful teaching process and repeatability. Moreover have to bear in mind that not all sclerotic process can be qualified as scleroderma. Therefore there are exclusions in new ACR/EULAR criteria, when skin thickening or hardening is due to other causes such as scarring after injury, trauma etc. and when there is no history of finger involvement, which enables to exclude other diseases such as generalized morphea. Other conditions mimicking SSc are, among others, eosinophilic fasciitis, scleroedema, scleromyxoedema, graft vs. host disease, diabetic cheiroarthropathy, and nephrogenic systemic fibrosis [1] (Table I).

Skin thickening is crucial to diagnose scleroderma.

\section{Skin thickening - skin thickening not due to scaring after trauma, injury}

\section{Sclerodactyly - 4 pts.}

The hallmark of the ACR/EULAR classification criteria concept is the accent put on the cardinal SSc manifestation - skin fibrosis of the hands. Based on the extent of skin fibrosis SSc is subdivided into limited (ICSSc) and diffuse cutaneous SSc (dcSSc). In IcSSc skin sclerosis is restricted to distal portion of the limbs, including hands, but also to the face, whereas in dcSSc the skin involvement is extending proximal to the elbows and knees.

Most SSc patients have sclerodactyly, however, an individual may or may not have more extensive cutaneous involvement.

\section{Puffy fingers -2 pts.}

Puffy fingers (or puffy hands; PuFy), defined as the increase of soft tissue mass of the fingers extending beyond the confines of the joint capsule, are regarded as an early indicator of connective tissue diseases. This 


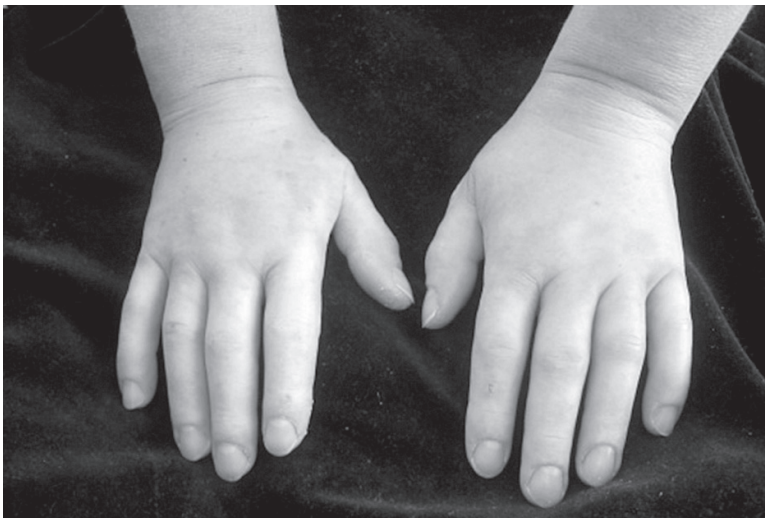

Fig. 1. Puffy fingers (PuFy).

fairly sudden onset swelling of hands has been identified as an independent factor of SSc development in patients with RP [12]. According to the opinion of a large number of scleroderma experts who were engaged in a multicenter Delphi exercise conducted by EULAR and EUSTAR, the occurrence of puffy fingers is one of three red flags that should arise concern of the very early SSC.

A number of studies evaluated the significance of PuFy in the transition into SSc [13]. In VEDOSS (Very Early Diagnosis Of Systemic Sclerosis) study [14], up to February 2012, 469 patients presenting RP were enrolled in order to assess the prevalence of the diagnostic items identified by EULAR as preliminary criteria for very early diagnosis of SSC, including the puffy fingers. Previous or current puffy fingers were predominantly found in ANA (antinuclear antibody) - positive patients (38.5\% vs. $23.5 \%$ in ANA negative group, $p=0.001$ ), and were the most frequent feature in this group. Additionally $10.5 \%$ of ANA + cases had sclerodactyly comparing to $4.9 \%$ in patients without ANA. In almost $60 \%$ of patients with previous or current PuFy the Ssc - specific autoantibodies were detected (ACA in 76.4\%, TOPO-I in 23.6\%). Moreover, the presence of the RP and ANA in PuFy population constitutes a red flag raising a suspicion of a very early SSc, and increased incidence of coexisting sclerodactyly, teleangiectases and oesophageal symptoms were noted. More than seventy percent of ANA + PuFy patients have also an SSc nailfold capillaroscopy (NC) pattern (compared to $41 \%$ in PuFy-negative group). Nearly $90 \%$ of patients from this group have also presented a SscNC pattern or/and Ssc - specific antibodies, and therefore could be classified as early systemic sclerosis using the VEDOSS criteria. The positive predictive value (PPV) of the progression to SSc in patients RP $(+)$ and ANA (+) with the puffy fingers is $88.5 \%$, compared to only $33.9 \%$ where there is no history of PuFy. Due to the absence of longitudinal observations, the negative predictive value was not determined (Fig. 1).

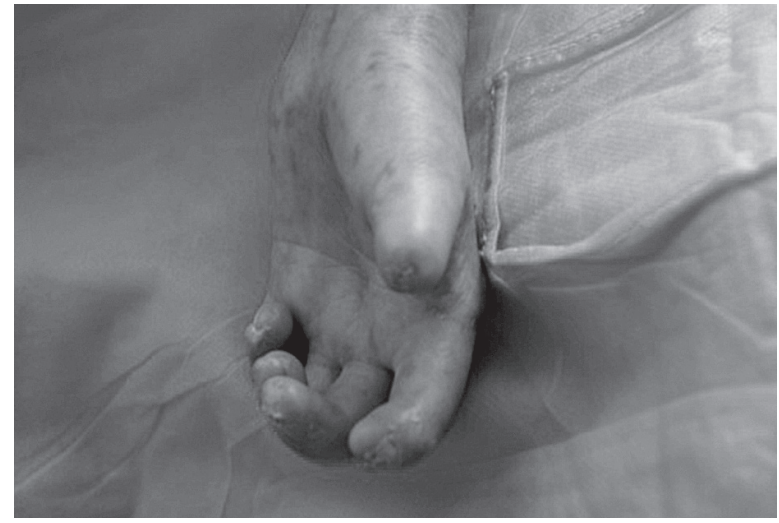

Fig. 2. Digital tip ulcer.

There are no established standards for assessing PuFy, hence the occurrence of PuFy should be confirmed by an expert, and simultaneously the causes such as edema or inflammatory dactylitis should be excluded.

Presence of PuFy is a meaningful symptom, raising suspicion of a very early SSc in patients with RP.

\section{Digital tip ulcers -2 pts., Fingertip pitting scars -3 pts.}

This criterion concerns ulcer or scars distal or at PIP joints which occured regardless of trauma. Digital pitting scars appear as a result of ischemia and look like depressed area.

Recently published new data in pathogenic changes in scleroderma showed that early vascular events include endothelium's dysfunction and injury with apoptosis of endothelial cells. Avouac et al. [8] have proven a role of the vascular endothelial growth factor (VEGF) in SSc-vasculopathy. VEGF dose was connected with strong profibrotic effects. Moreover, profibrotic effects were accompanied by a vasculopathy with an increase in vessel wall thickness, which is a classical feature of SSc-microangiopathy. In the VEDOSS study showed that digital lesions, in particular digital ulcers are associated with internal organ, especially gastrointestinal involvement [15].

Digital tip ulcer and pitting scars appeared in 53\% in derivation sample and in 40\%,39\%, respectively, in validation sample. In both groups data were statistically significant from scleroderma like disorder (Fig. 2).

\section{Telangiectasia - 2 pts.}

Telangiectasia, which result from vasculopathy, are visible dilated superficial blood vessels, often found in face, lips, hand and inside the mouth. They should be distinguished from spider angiomas with central arteriole. The authors of 230 SSc EUSTAR patients research 


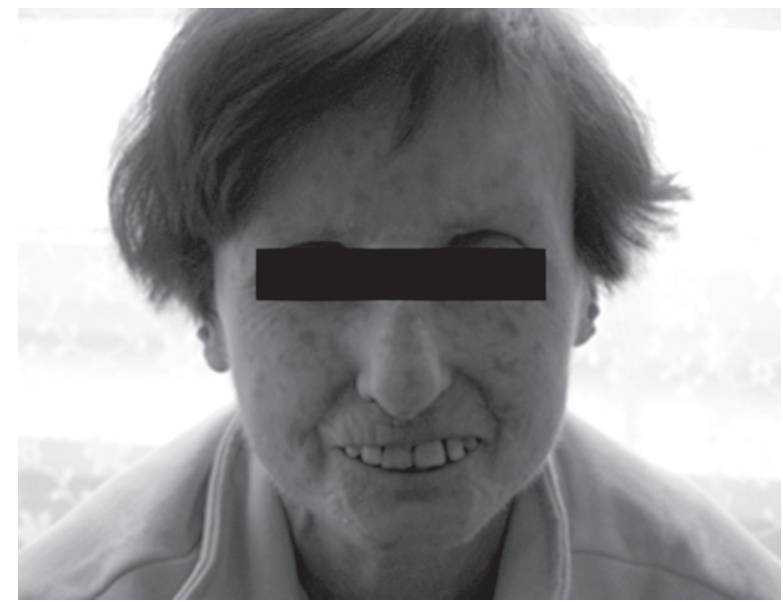

Fig. 3. Teleangiectasia.

group from Peking Union Medical College Hospital have found that telangiectasia could be clinical marker of microvascular disease in SSc. RP (97.9\% vs. 90.3\%), digital ulcers (DUs; $40.6 \%$ vs. 23.1\%) and digital pitting (49.0\% vs. $33.8 \%$ ) were significantly more frequent in telangiectasia patients $(p<0.05)[16]$.

Telangiectasia appeared in 35\% in derivation sample and in $25 \%$ in validation sample in both cases the data were statistically significant for scleroderma like disorder (Fig. 3) [1].

\section{Raynaud's phenomenon (RP) - 3 pts.}

Raynaud's phenomenon - is a common clinical symptom, affecting 3-5\% of the population. When the aetiology is unknown, as there is no other disease associated, the disorder is classified as primary and can be referred to as Raynaud's disease [17]. RP is considered secondary, when it occurs in association with an underlying disease, most frequently CTD. The majority of cases are due to primary RP and a reliable feature to differentiate between those two is the presence of $a b$ normal capillaries in a NC. Based on the observation of 569 patients with isolated CTD with negative ANA and normal NC, Moinzadeh et al. [18] reported no progression to CTD in $95 \%$ of cases.

$\mathrm{RP}$ is characterized by episodic, reversible vasospasm of the peripheral arteries in fingers and often in toes, in response to temperature changes, beta blockers, smoking or emotional stress. Although the classical progression consists of 3 phases, usually the episodes involve only the two of them. Typically it is pallor, followed by cyanosis and finally rubor whilst rewarming. The cyanosis, which is an effect of deoxygenation, occurs only in the most severe cases. It is generally accepted that the diagnosis of RP is based on the history of at least 2 colour changes reported by a physician or the patient alone. In this approach, patients with only the ischemic phase are excluded. However, Ingegnoli et al. [19] strongly suggest not to prevent such patients from further evaluation, as according to the study, $27 \%$ of them were diagnosed or suspected of CTD.

From clinical point of view, for early identification of SSc RP offers several advantages. It occurs in more than $95 \%$ of patients with SSc [20]. It is also regarded as one of the earliest symptoms of SSc development, preceding the skin and internal organs fibrosis by years or decades. The incidence of developing a definite SSc among patients with an isolated RP was assessed in a number of studies and summarized in a meta-analysis by Spencer-Green [21]. The results are consistent with the observation from a recent large prospective study by Koening - $12.6 \%$. The median time between the first episode of RP and the diagnosis of definite SSc was 4.56 years [13].

Although the RP is present in all CTDs, it was included in the new 2013 classification criteria because the lack of RP in the course of SSc is so unusual, that it adds statistical value to the criteria. Moreover, despite the risk of over-diagnosis, it is important to identify the patients with very early stage disease, as the severe complications of lungs, kidneys, heart or gastrointestinal tract usually develop within the first 3 years of the disease.

\section{Abnormal nailfold capillaries - 2 pts.}

Nailfold capillaroscopy is a method showing importance for the evaluation of microcirculation in patients with RP. This imaging technique is easy to repeat, non-invasive and inexpensive. It has become a gold standard in diagnostic process of scleroderma. Raynaud's phenomenon remains a typical manifestation of vascular involvement in scleroderma. Nailfold capillaroscopy and immunological tests together with physical examination allow distinguishing primary RP from secondary.

Capillaroscopy is used in medicine since 1823, but only since 1973 when Maricq and Le Roy described the specific capillaroscopic pattern in systemic sclerosis, detection of abnormal capillaroscopic patterns has had high positive predictive value for the development of systemic rheumatic disease. A meta-analysis by Spencer-Green [21] showed a positive predictive value in 47\% for the presence of abnormal changes in capillaroscopy. At the same time the predictive value for the presence of autoantibodies is 30\% [21]. Additionally, when presence of specific autoantibodies in SSc is associated with abnormal capillaroscopy findings, the positive predictive value for the development of SSc in the next 15 years reaches $79.5 \%$ [13].

The specific capillaroscopic pattern is characterized by the presence of megacapillaries, micro-bleeding, loss of capillary loops, with consequent reduction in the num- 
ber of capillaries and also by neoangiogenesis [22]. Lately, Cutolo et al. [23] classified above-mentioned capillaroscopy changes in three stages: early, active and late.

The "early" pattern is characterized by micro-hemorrhages, megacapillaries and by no significant devascularization with a relatively preserved capillary distribution. These findings are crucial for early diagnosis of SSc.

In the "active" pattern an increased number of megacapillaries and micro-hemorrhages in association with a mild distortion of capillary architecture and moderate loss of capillaries can be observed.

The "late" pattern is described as a severe loss of capillaries and characterizes with avascular areas. Neoangiogenesis and disorganization of capillary architecture is also very characteristic [13].

What is more, Cutolo's et al. research confirmed an association between capillaroscopic changes and disease duration. The research also proved a correlation between different capillaroscopic patterns (early, active, late) and the presence of autoantibodies (anticentromere, anti-topoisomerase I), as well as cutaneous subtype: limited and diffuse [22, 23].

In the following years the role of capillaroscopy increased. Recent studies have proposed using capillaroscopy as clinical biomarker of disease activity and severity. Some studies even suggested an association between the grade of capillary dilation or capillary loss and SSc-related organ involvement [24, 25].

Several studies showed a correlation between capillaroscopic damage and lung involvement $[25,26]$. Bredemeier et al. [27] described an association between active lung involvement and the mean avascular score in patients whose disease duration was less than 5 years [27].

Moreover, Sebastiani et al. [28] have proven a significant association between ischemic lesions and the CSURI, a capillaroscopic skin ulcer risk index. A receiver operating characteristic curve analysis showed an area under the curve of 0.926 for ulcer appearance at the cut off value of 2.94 .

Lately Kayser et al. [29] observed that avascular score higher than 1.5 at capillaroscopy was an independent predictor of death in SSc, suggesting that capillaroscopy can be useful for predicting SSc outcome.

NC is an easy technique which is commonly accepted as a standard in the assessment of the microcirculatory pathologies due to characteristic SSc pattern.

\section{Scleroderma related antibodies (any of anti- centromere, anti-topoisomerase I, anti-Scl 70, anti-RNA polymerase III) -3 pts.}

Although the presence of autoantibodies is one of the characteristics of SSc, there is no single laboratory test to diagnose SSc. The autoantibodies occur in 90-
95\% of patients with SSc and their profile has a predictive value for the prognosis. However, there is a distinct subset of ANA negative patients, which beside undeniable identifiable features such as less intensity of vasculopathic manifestations, does not differ in all-cause mortality [30].

It is generally accepted that anti-topo-I and antiCENP-B are the predictors of progression from isolated RP to SSc [2]. A twenty-year prospective study of 586 patients with RP examined the influence of the major SSc - specific antibodies, as well as the SSc pattern NC, on the progression to definite Ssc. The study demonstrates that the presence of anti-CENP-B is an independent risk factor of enlarged capillaries. Moreover, the study also revealed that RNAP III are associated with greater risk of capillary loss. In a French Canadian population of patients with RP who later developed an overt SSc, the Ssc specific ANA were observed in $78.4 \%$ at baseline. The most common was anti-CENP-B (44.6\%). Overall, among the patients with RP and Ssc-specific antibodies, the progression to definite SsC was reported in $35.4 \%$ cases. Based on the above, the presence of Ssc-specific autoantibodies in RP patients is related with an 8 -fold increased risk of developing definite Ssc [11].

The widely available Ssc-specific ANA such as anticentromere and anti-topoisomerase I, were also proposed, next to NC, in VEDOSS criteria, as necessary to confirm a high feasibility of very early Ssc. An important point to make, the 2013 criteria included patients from Europe and North America, while the distribution of autoantibodies vary between different countries. At the moment, the additional Ssc-antibodies such as anti-Th/ To, anti-U3-RNP are not commonly available.

The new classification criteria include the significant progress made in the diagnostic of SSc, incorporating the specific serum autoantibodies as one of the items. In order to meet the SSc-related autoantibodies criterion, at least one of the anticentromere, anti-topoisomerase I, anti-RNA-polimerase III has to be positive, according to local laboratory standards. The method of antibody testing was not suggested.

\section{Pulmonary arterial hypertension - 2 pts.}

Pulmonary arterial hypertension (PAH) is prevalent in around 9\% of patients with connective tissue diseases, such as patients with systemic sclerosis (SSc). PAH is connected with well-established scleroderma and visceral involvement.

Twenty-six percent of SSc patients died because of PAH. In 2015 ESC (The European Society of Cardiology) recommended $\mathrm{PAH}$ guidelines with Scleroderma patients [31]. Criteria for PAH have changed over the years 
and the diagnosis of PAH should be based on the most recent accepted criteria from right heart catheterization. Therefore, the gold standard for the diagnosis of PAH is right heart catheterization (RHC), while screening methods should use tools that are non-invasive, reproducible, associated with a high negative predictive value for the condition and cost effective. In PAH, these tools include pulmonary function tests (PFTs), circulating biomarkers and echocardiography. First recommendation concerned resting echocardiography which is a screening test in asymptomatic patients with systemic sclerosis I B [32].

In SSc, PFTs have long been used as a screening tool like diffusing capacity of the lung for carbon monoxide (DLCO) [31, 32]. An increased risk of PAH has been shown in adult SSc patients with a DLCO $60 \%$ of predicted value [33].

Recent studies confirmed that biomarkers [N-terminal pro-brain natriuretic peptide (NT-proBNP)], alone $[34,35]$ or in combination with PFTs, may identify patients at higher risk to present SSc-PAH $[36,37]$.

Moreover, systemic sclerosis patients with a mean PAP ranging from 21 to $24 \mathrm{~mm} \mathrm{Hg}$ should be closely monitored, because of a higher risk of PAH [30]. Initial screening using the stepwise DETECT algorithm may be considered in adult systemic sclerosis patients with $>3$ years' disease duration and lower DLCO [30].

Many researches in SSc have proven that asymptomatic PAH patients detected by screening can be missed by Doppler echocardiography, what requests a need for a multitest approach [31].

Avouac et al. [38] presented an expert consensus, using Delphi method, to identify which criteria are the most appropriate in clinical practice to refer SSc-patients for RHC. Experts selected criteria based on clinical assessment as follows: unexplained dyspnea, progressive dyspnea over the past 3 months, worsening of WHO dyspnea functional class, any finding on physical examination suggestive of elevated right heart pressures and any sign of right heart failure. In echocardiography systolic pulmonary artery pressure [PAPS] $>45 \mathrm{~mm} \mathrm{Hg}$ and right ventricle dilation were distinguished. In pulmonary function tests were marked DLCO < $60 \%$ without pulmonary fibrosis [PF].

For PAH detection in SSc, the DETECT algorithm has been written which included ACA positivity, the predicted FVC\%/DLCO\% ratio and the presence of current or past teleangiectasia, NT-proBNP levels, urate levels and a right axis deviation on ECG and echographic parameters (right atrium area and tricuspid regurgitant jet velocity) [34, 39]. In this study, adult SSc patients with 3 -years disease duration and a DLCO of $60 \%$ of predicted value underwent non-invasive testing and RHC. After assessment for six clinical plausibility and feasibil- ity, these were incorporated into a two-step, internally validated detection algorithm. Step 1 of the algorithm determined referral to echocardiography. In step 2, the step 1 prediction score and two echocardiographic variables determined referral to RHC. The DETECT algorithm recommended $\mathrm{RHC}$ in $62 \%$ of patients (referral rate) and missed $4 \%$ of PAH patients (false negatives). Of those, $19 \%$ had RHC-confirmed PAH. A novel evidence-based DETECT algorithm for PAH detection in SSc is proposed as a sensitive, non-invasive tool which minimizes missed diagnoses, identifies milder disease and addresses resource usage [34].

For PAH detection in SSc, the DETECT algorithm included:

- ACA positivity,

- the predicted FVC\%/DLCO\% ratio,

- the presence of current or past teleangiectasia,

- NT-proBNP levels,

- urate levels,

- a right axis deviation on ECG,

- echographic parameters (right atrium area and tricuspid regurgitant jet velocity).

\section{Interstitial lung disease $\mathbf{- 2}$ pts.}

Interstitial lung disease (ILD) is one of the most common manifestations of CTD, with a broad spectrum of associated clinical entities. Interstitial lung disease is particularly common is SSC [40]. However, lung involvement may also occur in rheumatoid arthritis, mixed connective tissue disease, polymyositis or dermatomyositis and Sjögren syndrome, as well as in systemic lupus erythematosus, although it is unusual. Interstitial lung disease can occur in patients with a definite CTD, however, it can also be the first manifestation of the disease. It is important to note that, according to Bruni and colleagues, a quarter of patients who did not meet the 1980 criteria of SSc may already have ILD [41]. Valentini et al. [42] studied the organ involvement in patients with early SSc, defined as RP with SSc-specific autoantibodies or/and typical Ssc NC pattern with no other symptoms than puffy fingers and arthritis. They proved, that a decrease in DLCO $<80 \%$ can be seen in nearly a third of those patients. Based on autopsy observations, D'Angelo reported that fibrosis was present in more than $70 \%$ of the Ssc patients [43]. ILD may be confirmed in several ways e.g. by a chest radiograph or by a gold standard that is a computed tomography. A thoracoscopic or open lung biopsy is usually not performed for diagnosis of SSc-ILD. At this point, worth mentioning is the study of Legnani [44], measuring the lung carbon monoxide diffusion during effort in SSc patients. The data suggest that exertional DLCO may reveal latent DLCO impairment. This 
observation can be helpful to detect lung damage earlier than DLCO at rest. Nevertheless, due to little added value, diffusing capacity and forced vital capacity were excluded through the process of developing the new classification criteria.

The new 2013 classification criteria include interstitial lung disease, comprising pulmonary fibrosis most evident in the basilar parts of the lungs as well as the presence of "Velcro" crackles on auscultation, whereas no other cause such as congestive heart failure is suspected.

\section{Scleroderma renal crisis}

Several items, which are useful in clinical practice in recognizing SSc, among others, renal crisis, flexion contractures of the fingers, calcinosis, tendon or bursal friction rubs, esophageal dilatation and dysphagia, are not included in the new ACR/EULAR criteria. After consideration, the above cited criteria did not substantially improve sensitivity or specificity. For example, renal crisis is a strong indicator of SSc, but its low occurrence makes it less useful for the purpose of classification.

Scleroderma renal crisis (SRC), which is characterized by sudden severe hypertension, acute renal injury with elevated creatinine and intravascular haemolysis, is a potentially lethal clinical manifestation if not recognized. However, despite the reports of the frequency of $12-18 \%$ before 1970 , its prevalence has been recently estimated in the EUSTAR cohort at 2\% [45]. The onset is more commonly observed in male patients, early after the diagnosis of dsSSc, especially those with a rapid progressive skin fibrosis. A well-documented risk factor is the presence of anti-RNA - polymerase III antibodies and exposure to corticosteroids (especially high dose) $[46,47]$.

SRC was considered as an item for the new 2013 criteria, however, due to its low frequency it did not relevantly improve neither sensitivity nor specificity.

\section{Summary}

The cooperation of European and American rheumatic associations is essential for reaching the consensus in the diagnosis and treatment in orphan diseases such as scleroderma.

The ACR-EULAR classification criteria for SSc perform better in the studies than 1980 Preliminary ARA Criteria for SSc both in terms of sensitivity and specificity. They are better when applied to individual subjects and also may classify the patients in both early and in late stage of disease. The criteria take into account the organ involvement and immunological profile of SSc patients. Some of symptoms e.g. renal crisis or esoph- ageal involvement (dysphagia and reflux) or calcinosis were not included in this criteria. But for clinicians these symptoms can be a valuable clue, however, according to the authors of the criteria their inclusion does not significantly improved sensitivity and specificity of the SSC criteria [1]. Undoubtedly many studies over the last three years confirmed usefulness of new ACR/ EULAR criteria in clinical practice.

The authors declare no conflict of interest.

\section{References}

1. Hoogen F, Khanna D, Johnson SR, et al. Classification criteria for systemic sclerosis: an American College of Rheumatology/European League against Rheumatism collaborative initiative. Arthritis Rheum 2013; 65: 2737-2747.

2. Jordan S, Maurer B, Toniolo M, et al. Performance of the new ACR/EULAR classification criteria for systemic sclerosis in clinical practice. Rheumatology (Oxford) 2015; 54: 1454-1458.

3. Alhajeri H, Hudson M, Fritzler M, et al. 2013 American College of Rheumatology/European League against rheumatism classification criteria for systemic sclerosis outperform the 1980 criteria: data from the Canadian Scleroderma Research Group. Arthritis Care Res (Hoboken) 2015; 67: 582-587.

4. Aggarwal R, Ringold S, Khanna D, et al. Distinctions between diagnostic and classification criteria? Arthritis Care Res (Hoboken) 2015; 67: 891-897.

5. Lonzetti LS, Joyal F, Raynauld JP, et al. Updating the American College of Rheumatology preliminary classification criteria for systemic sclerosis: addition of severe nailfold capillaroscopy abnormalities markedly increases the sensitivity for limited scleroderma. Arthritis Rheum 2001; 44: 735-736.

6. Nadashkevich O, Davis P, Fritzler MJ. A proposal of criteria for the classification of systemic sclerosis. Med Sci Monit 2004; 10: CR615-621.

7. LeRoy EC, Medsger TA Jr. Criteria for the classification of early systemic sclerosis. J Rheumatol 2001; 28: 1573-1578.

8. Avouac J, Fransen J, Walker UA, et al. Preliminary criteria for the very early diagnosis of systemic sclerosis: results of a Delphi Consensus Study from EULAR Scleroderma Trials and Research Group. Ann Rheum Dis 2011; 70: 476-481.

9. Clements PJ1, Hurwitz EL, Wong WK, et al. Skin thickness score as a predictor and correlate of outcome in systemic sclerosis: high-dose versus low-dose penicillamine trial. Arthritis Rheum 2000; 43: 2445-2454.

10. Shand L, Lunt M. Relationship Between Change in Skin Score and Disease Outcome in Diffuse Cutaneous Systemic Sclerosis. Arthritis Rheumatism 2007; 56: 2422-2431.

11. Czirják L, Foeldvari I, Müller-Ladner U. Skin involvement in systemic sclerosis. Rheumatology (Oxford) 2008; 47 Suppl 5: v44-45.

12. Gerbracht DD, Steen VD, Ziegler GL, et al. Evolution of primary Raynaud's phenomenon (Raynaud's disease) to connective tissue disease. Arthritis Rheum 1985; 28: 87-92. 
13. Koenig M, Joyal F, Fritzler MJ, et al. Autoantibodies and microvascular damage are independent predictive factors for the progression of Raynaud's phenomenon to systemic sclerosis: a twenty-year prospective study of 586 patients, with validation of proposed criteria for early systemic sclerosis. Arthritis Rheum 2008; 58: 3902-3912.

14. Minier T, Guiducci S, Bellando-Randone S, et al. Preliminary analysis of the Very Early Diagnosis of Systemic Sclerosis (VEDOSS) EUSTAR multicentre study: evidence for puffy fingers as a pivotal sign for suspicion of systemic sclerosis. Ann Rheum Dis 2014; 73: 2087-2093.

15. Bruni C, Guiducci S, Bellando-Randone S, et al. Digital ulcers as a sentinel sign for early internal organ involvement in very early systemic sclerosis. Rheumatology (Oxford) 2015; 54: 72 76.

16. Zhang SZ, Xu D, Li MT, et al. Telangiectasia as a potential clinical marker of microvascular lesions in systemic sclerosis patients from EUSTAR data in China. Clin Exp Rheumatol 2015, 33 (4 Suppl 91): S106-110.

17. Pope JE. The Diagnosis and Treatment of Raynaud's Phenomenon. A Practical Approach. Drugs 2007; 67: 517-525.

18. Moinzadeh P, Howell KJ, Ong V, et al. Progression of Isolated Raynaud's Phenomenon to Systemic Sclerosis: Impact of ANA Subtype And Disease Subset [abstract]. Arthritis Rheum 2011; 63 Suppl 10: 677.

19. Ingegnoli F, Gualtierotti R, Orenti A, et al. Uniphasic Blanching of the Fingers, Abnormal Capillaroscopy in Nonsymptomatic Digits, and Autoantibodies: Expanding Options to Increase the Level of Suspicion of Connective Tissue Diseases beyond the Classification of Raynaud's Phenomenon. J Immunol Res 2015; Article ID 371960.

20. Valentini G, Vettori S, Cuomo G, et al. Early systemic sclerosis: short-term disease evolution and factors predicting the development of new manifestations of organ involvement. Arthritis Res Ther 2012; 4: R188.

21. Spencer-Green G. Outcomes in primary Raynaud phenomenon: a metaanalysis of the frequency, rates, and predictors of transition to secondary diseases. Arch Intern Med 1998; 158: 595-600.

22. Herrick AL, Cutolo M. Clinical implications from capillaroscopic analysis in patients with Raynaud's phenomenon and systemic sclerosis. Arthritis Rheum 2010; 62: 2595-2604.

23. Cutolo M, Pizzorni C, Tuccio $M$, et al. Nailfold videocapillaroscopic patterns and serum autoantibodies in systemic sclerosis. Rheumatology (Oxford) 2004; 43: 719-726.

24. Caramaschi P, Canestrini S, Martinelli N, et al. Scleroderma patients nailfold videocapillaroscopic patterns are associated with disease subset and disease severity. Rheumatology (Oxford) 2007; 46: 1566-1569.

25. Pucinelli ML, Atra E, Sato El, et al. Nailfold capillaroscopy in systemic sclerosis: correlations with involvement of lung and esophagus. Rev Bras Rheumatol 1995; 35: 136-142.

26. Groen H, Wichers G, ter Borg EJ, et al. Pulmonary diffusing capacity disturbances are related to nailfold capillaroscopy changes in patients with Raynaudis phenomenon with and without an underlying connective tissue disease. Am J Med 1990; 89: 34-41.
27. Bredemeier M, Xavier RM, Capobianco KG, et al. Nailfold capillary microscopy can suggest pulmonary disease activity in systemic sclerosis. J Rheumatol 2004; 31: 286-294.

28. Sebastiani M, Manfredi A, Colaci M, et al. Capillaroscopic skin ulcer risk index: a new prognostic tool for digital skin ulcer development in systemic sclerosis patients. Arthritis Rheum 2009; 61: 688-694.

29. Kayser C1, Sekiyama JY, Próspero LC, et al. Nailfold capillaroscopy abnormalities as predictors of mortality in patients with systemic sclerosis. Clin Exp Rheumatol 2013; 31 (2 Suppl 76): 103-108.

30. Salazar GA, Assassi S, Wigley F, et al. Antinuclear antibody-negative systemic sclerosis. Semin Arthritis Rheum 2015; 44 : 680-686.

31. 2015 ESC/ERS Guidelines for the diagnosis and treatment of pulmonary hypertension European Heart Journal doi:10.1093/ eurheartj/ehv317

32. Steen V, Medsger TA. Predictors of isolated pulmonary hypertension in patients with systemic sclerosis and limited cutaneous involvement. Arthritis Rheum 2003; 48: 516-522.

33. Schreiber BE, Valerio CJ, Keir GJ, et al. Improving the detection of pulmonary hypertension in systemic sclerosis using pulmonary function tests. Arthritis Rheum 2011; 63: 3531-3539.

34. Coghlan JG, Denton CP, Grunig E, et al. Evidence-based detection of pulmonary arterial hypertension in systemic sclerosis: the DETECT study. Ann Rheum Dis 2014; 73: 1340-1349.

35. Williams MH, Handler CE, Akram R, et al. Role of N-terminal brain natriuretic peptide (N-TproBNP) in scleroderma-associated pulmonary arterial hypertension. Eur Heart J 2006; 27: 1485-1494.

36. Cavagna L, Caporali R, Klersy C, et al. Comparison of brain natriuretic peptide (BNP) and NT-proBNP in screening for pulmonary arterial hypertension in patients with systemic sclerosis. J Rheumatol 2010; 37: 2064-2070.

37. Allanore Y, Borderie D, Avouac J, et al. High N-terminal probrain natriuretic peptide levels and low diffusing capacity for carbon monoxide as independent predictors of the occurrence of precapillary pulmonary arterial hypertension in patients with systemic sclerosis. Arthritis Rheum 2008; 58: 284-291.

38. Avouac J, Huscher D, Furst DE, et al. Expert consensus for performing right heart catheterisation for suspected pulmonary arterial hypertension in systemic sclerosis: a Delphi consensus study with cluster analysis. Ann Rheum Dis 2014; 73 : 191-197.

39. http://detect-pah.com/ access: 08.11.2016

40. Bouros D, Wells AU, Nicholson AG, et al. Histopathologic subsets of fibrosing alveolitis in patients with systemic sclerosis and their relationship to outcome. Am J Respir Crit Care Med 2002; 165: 1581-1586.

41. Bruni C, Guiducci S, Bellando-Randone S, et al. Digital ulcers as a sentinel sign for early internalorgan involvement in very early systemic sclerosis. Rheumatology 2015; 54: 72-76.

42. Valentini G, Marcoccia A, Cuomo G. Early systemic sclerosis: analysis of the disease course in patients with marker autoantibody and/or capillaroscopic positivity. Arthritis Care Res (Hoboken) 2014; 66: 1520-1527.

43. D’Angelo WA, Fries JF, Masi AT, Shulman LE. Pathologic observations in systemic sclerosis (scleroderma). A study of fif- 
ty-eight autopsy cases and fifty-eight matched controls. Am J Med 1969; 46: 428-440.

44. Legnani D, Rizzi M, Sarzi-Puttini P, et al. Diffusing Pulmonary Capacity Measured During Effort: A Possible Early Marker of Pulmonary Involvement In Systemic Sclerosis. Isr Med Assoc J 2015; 17: 739-743.

45. Allanore Y, Meune C, Vonk MC, et al. Prevalence and factors associated with left ventricular dysfunctionin the EULAR Scleroderma Trial and Research group (EUSTAR) database of patients with systemic sclerosis. Ann Rheum Dis 2010; 69: 218-221.

46. Lee JJ, Pope JE. Diagnosis and Management of Systemic Sclerosis: A Practical Approach. Drugs 2016; 76: 203-213.

47. Castro SV, Jimenez SA. Biomarkers in systemic sclerosis. Biomark Med 2010; 4: 133-147. 